\title{
Adverse cognitive effect of gabapentin in individuals with spinal cord injury: preliminary findings
}

\author{
Kazuko Shem $^{1} \cdot$ Steven Barncord ${ }^{2} \cdot$ Kara Flavin $^{3} \cdot$ Manoj Mohan $^{4}$
}

Received: 15 October 2017 / Revised: 23 December 2017 / Accepted: 23 December 2017

(c) International Spinal Cord Society 2018

\begin{abstract}
Study design Prospective observational cohort study.

Objectives To assess the effects of gabapentin on neuropsychological variables including memory, attention, and executive function in individuals with spinal cord injury.

Setting Santa Clara Valley Medical Center inpatient spinal cord injury unit.

Methods Ten patients (three females, seven males) with traumatic spinal cord injury underwent testing, with a mean age of 35.6 years (range $19-59, \mathrm{SD} \pm 15.74$ ). There were five patients with tetraplegia and five with paraplegia. Nine tests to assess neuropsychological function and two tests to assess pain were performed at 1 week post initiation of therapy, and at 4 weeks post initiation of therapy. The neuropsychological tests assessed aspects of memory, attention, and executive function.

Results The average score for six out of the nine neuropsychological items administered at 1 week post initiation of gabapentin displayed a decrease in cognitive function when compared to baseline. The average score for five out of nine neuropsychological items improved from 1 week post initiation of treatment to 4 weeks post initiation of treatment.

Conclusions Gabapentin therapy is associated with a tangible decline in memory, executive function, and attention in individuals with spinal cord injury. However, owing to small sample size, loss of patient follow-up at the 4 week posttreatment assessment, and lack of a control group, we cannot definitively state that any decreases in cognition are solely attributable to treatment with gabapentin.
\end{abstract}

\section{Introduction}

Acute and chronic pain are common and well-described consequences of spinal cord injury (SCI) that are consistently reported to occur in over $70 \%$ of patients [1-3]. Chronic pain is defined as pain lasting longer than 12 weeks, and has been found to be associated with more depressive symptoms, more perceived stress, and poorer

Kazuko Shem

kazuko.shem@hhs.sccgov.org

1 Department of Physical Medicine and Rehabilitation, Santa Clara Valley Medical Center, San Jose, CA, USA

2 Department of Psychiatry, Santa Clara Valley Medical Center, San Jose, CA, USA

3 Division of Physical Medicine and Rehabilitation, Stanford Healthcare, Palo Alto, CA, USA

4 Department of Physical Medicine and Rehabilitation, University of Pittsburgh Medical Center, Pittsburgh, PA, USA self-assessed health in individuals with SCI [1]. Neuropathic pain is a subtype of acute and chronic pain that is prevalent in SCI, affecting from 38 to $77 \%$ of patients [46].

Neuropathic pain is defined by the International Association for the Study of Pain (IASP) Special Interest Group on Neuropathic Pain as "pain arising as a direct consequence of a lesion or a disease affecting the somatosensory system" [7]. The clinical manifestation of neuropathic pain varies, but has been summarized as "a sensory deficit and the presence of paradoxical pain in the same region", leading to allodynia, hyperalgesia, paroxysms, paresthesias, and dysesthesias in the affected area(s) [8]. The cause of neuropathic pain is unknown, but some hypotheses suggest it is due to increased excitatory glutaminergic activity, which leads to upregulation of neuronal excitability and loss of endogenous inhibition [9].

Conventional pain medications, such as non-steroidal anti-inflammatory medications and opioids, are less effective for the treatment of neuropathic pain and also have subjective side-effects including decreased arousal and 
difficulty maintaining attention [10]. Gabapentin, an anticonvulsant, is generally regarded as a first line treatment for neuropathic pain though its mechanism of action is not completely understood [10]. It is hypothesized that gabapentin increases the concentration of GABA in the brain and reduces the release of several monoamine neurotransmitters [11]. The pharmacokinetics of gabapentin vary widely among patients, but the average time to reaching mean maximum plasma concentration is $2-3 \mathrm{~h}$ following a $300 \mathrm{mg}$ dose, with a drug half-life of 5-7 h [11]. Multiple studies have found gabapentin to be effective in reducing the severity and frequency of neuropathic pain in patients with both traumatic and non-traumatic SCI [12-16]; however, several studies have shown that gabapentin may have a negative effect on cognition [17-21].

The purpose of this study was to assess the effects of gabapentin on cognitive domains in individuals with traumatic SCI. For this study, we chose specific neuropsychological tests to isolate a subset of cognitive domains including attention, executive function, and memory. We hypothesized that if patients admitted to inpatient rehabilitation for SCI were administered gabapentin daily, they would experience a decrease in overall cognitive performance at the end of the first week, with a return to baseline during assessment at 4 weeks post treatment.

\section{Methods}

This study was approved by the Institutional Review Board of the medical center, and was a prospective, observational cohort study in individuals with traumatic SCI who were prescribed gabapentin for the symptoms of neuropathic pain during inpatient rehabilitation. When describing the neurologic status of each patient, the ASIA Impairment Scale was employed. Epidemiological data such as age, gender, time since injury, and level of injury were recorded. Data regarding gabapentin use including initial dose, final therapeutic dose, duration of use, and, if indicated, reason for cessation of use was collected. Neuropsychological tests were administered prior to initiation of gabapentin therapy and then re-administered after 1 week and 4 weeks post initiation of therapy. Pain level and pain interference with normal activity were assessed at the same time using the Brief Pain Inventory (BPI short form) [22].

Potential subjects were excluded if they were over the age of 70 because the prevalence of dementia has been found to be $13.9 \%$, which could confound the cognitive outcome measures of our study [23]. Patients under the age of 18 were also excluded, as their status as minors would have required parental consent at each stage of evaluation, which may not have been feasible during their hospital stay. Subjects were excluded if they had: an inability to speak
English, less than a 6th grade education (the 6th grade education or reading level is usually considered as a minimum to ensure people understand the direction and/or questions), concomitant brain injury (ruled out through neurologic exam, imaging review, and self reporting), significant cognitive impairment that would have interfered with the subject's ability to complete the neuropsychological testing, pregnancy, seizure disorder, the use of tricyclic antidepressants or other anticonvulsants, major depressive disorder (via self reporting), significant hepatic or renal insufficiency, diabetes mellitus, history of peripheral neuropathy, medical and psychiatric instability judged by the treating physician, prior history of sleep apnea, multiple sclerosis, or Guillain Barre syndrome.

The 11 measures used included Digit Span Forwards and Digit Span Backwards [24], Mental Control [24], Stroop Test [25], Word List for Immediate Recall and Word List for Delayed Recall [26], Controlled Oral Word Association Test [27], Oral Trail Making Test [28], Letter-Number Sequencing [29], and Pain Level and Pain Interference with Activity (both measured using Brief Pain Inventory Short Form) [29]. The three main aspects of cognitive function we attempted to isolate were executive function, memory, and attention. It should be noted that some of the neuropsychological tests assessed more than one cognitive function, resulting in an overlap in assessed cognitive domain among the various tests. In addition, Pain Level and the Pain Interference with Activity level as measured by the BPI were monitored concurrently with the other variables at one week and four-week post initiation of gabapentin. Please refer to Table 1 for information about administration of each test and the respective cognitive domain(s) assessed.

Instruments that could be administered without the use of upper extremities were chosen with attention to the robustness of their sensitivity, reliability, standardization, size of normative base, and wide-spread usage in the assessment community. All instruments were administered and scored in accordance with the providers' administration manuals or instructions. Because of the small sample size in the study and loss to follow-up of four patients, a comparison of demographic information such as age, gender and level of injury, and concomitant use of other pain medications with the neuropsychological results was not done.

\section{Results}

Ten individuals (three females, seven males) with SCI undergoing inpatient acute rehabilitation, who started gabapentin for the treatment of neuropathic pain, were prospectively included in this study. The mean age was 35.6 years (range 19-59, SD = 15.74). The ethnicities of the subjects were six Caucasians and four Hispanics. Five of the 


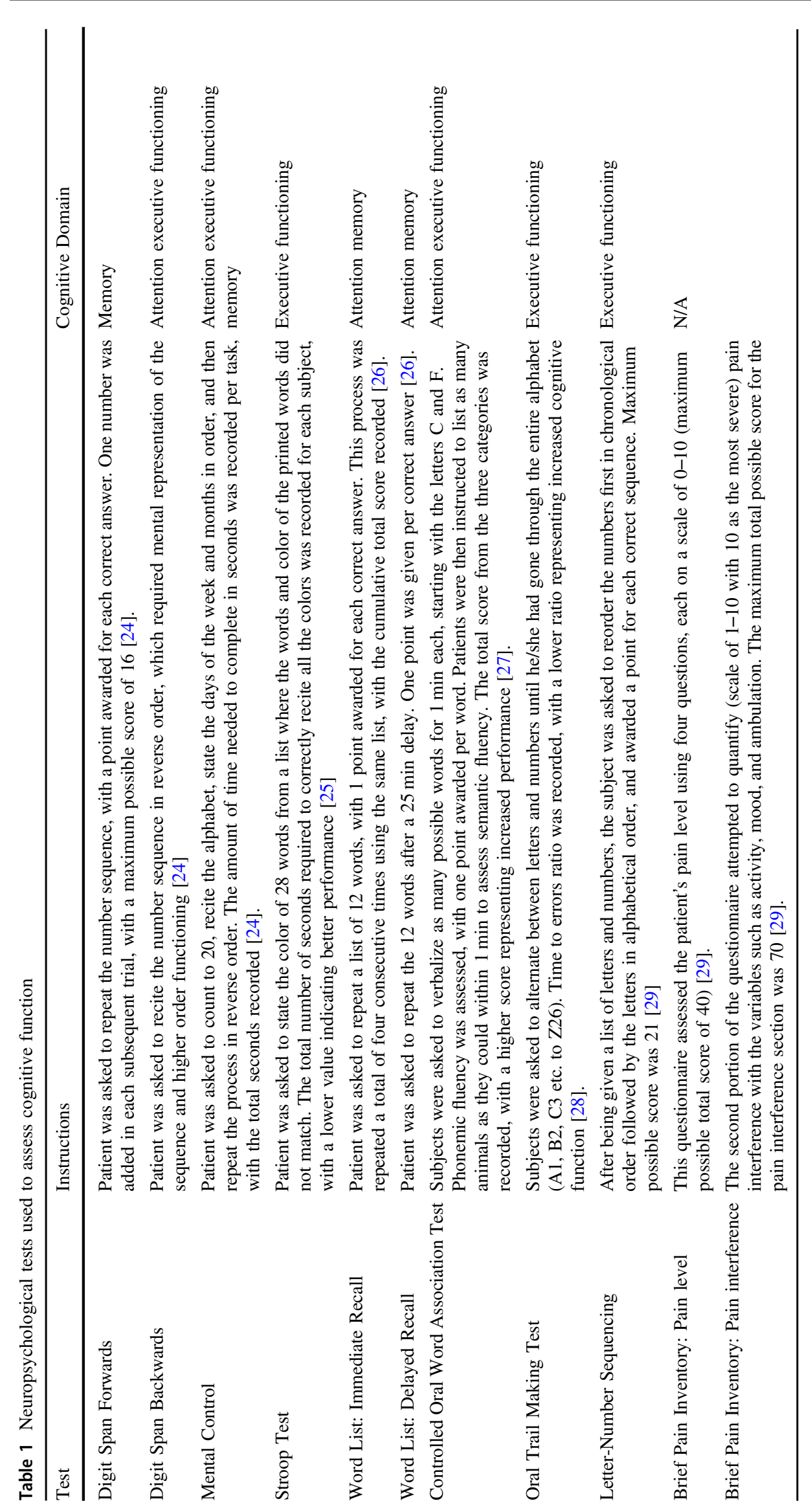


Table 2 Patient demographic information

\begin{tabular}{ll} 
Number of patients $^{\mathrm{a}}$ & 10 \\
Average patient age (SD) & $35.6(15.74)$ \\
Number of males & 7 \\
Number of females & 3 \\
Number of patients with tetraplegia & 5 \\
Number of patients with paraplegia & 5 \\
Average days since injury (SD) & 32.5 (37.4) \\
\hline a Four patients were lost to follow-up after the 1 week assessment \\
${ }^{\mathrm{b}}$ Values calculated based on nine patients, as one of the patient's time \\
since injury was over 4 years and treated as an outlier
\end{tabular}

participants had tetraplegia and five had paraplegia. There were four persons with ASIA A SCI, two with ASIA B, two with ASIA C, and two with ASIA D SCI. The etiologies of SCI were: motor vehicle accident $(n=4)$, gunshot wound $(n=3)$, fall $(n=1)$, sports $(n=1)$, and other trauma $(n=1)$ (Table 2).

The mean starting dose for gabapentin was $300 \mathrm{mg}$. The mean daily gabapentin dose was $1163 \mathrm{mg}(\mathrm{SD} \pm 994 \mathrm{mg})$ at week one (range 300-2100 mg) and $2340 \mathrm{mg}(\mathrm{SD} \pm 910$ $\mathrm{mg}$ ) during weeks 2 and 3 (range 1200-3600 mg). The dosage increases after week 1 were not initiated by the treating physicians, but by the patients if they felt their pain was not well controlled. All patients were taking various opioid medications such as morphine, oxycodone, tramadol, etc. during the study period. Given that all patients in this study had trauma and pain is prevalent in persons with SCI acutely, many patients were prescribed opioid medications by the surgeons prior to transferring to acute rehabilitation unit and were continued on them during rehabilitation.

Four out of 10 individuals completed only 1 week of this study. Three subjects were discharged from the hospital sooner than expected after the 1 week follow-up and one subject was discontinued on gabapentin by the treating physician as it was causing significant sedation and decline in cognitive function clinically. There were no statistical differences in pain level and level of pain interference between the patients who completed the study and those who dropped out.

The results of the neuropsychological tests are presented as following: a comparison of the baseline versus 1 week post treatment averages of the measured variables, and a comparison between the 1 and 4 week post treatment averages.

The results of the neuropsychological testing at baseline were compared with those of 1 week post initiation of gabapentin. Overall, the average scores for six out of the nine neuropsychological tests displayed decreased results during this period when compared with baseline values. The raw data and specific percentage changes of the neuropsychological test averages from baseline to 1 week post treatment and from 1 week post treatment to 4 weeks post treatment are displayed in Tables 3 and 4, respectively.

Of the neuropsychological measures most sensitive to attention, three out of the five test results displayed a decrease in cognitive function when assessed 1 week post treatment with gabapentin. Three out of the four neuropsychological measures sensitive to memory displayed decreases in cognitive function, with mental control as the variable that increased from baseline to 1 week post treatment. Three out of the six neuropsychological test averages sensitive to executive function showed a decrease (COWAT, Letter-Number Sequencing, and Oral Trail Making Test) at 1 week post treatment compared with baseline, while two out of the six (Mental control and Digit Span backwards) showed an increase in cognitive function. One test (Stroop test) remained unchanged 1 week post treatment with gabapentin.

A comparison of the 4 week post-treatment results to the 1 week post treatment scores revealed an increase in cognitive function in five out of nine neuropsychological variables. However, it is important to note that the results represent the average score of six patients, as four patients were lost to follow-up after the 1 week post treatment assessment. There was also a $22.75 \%$ increase in Pain Level and a $26.83 \%$ increase in Pain Interference with activity during this same interval. The average score for three out of the five neuropsychological measures sensitive to attention increased during this period. Three out of the four neuropsychological measures sensitive to testing memory displayed an increase as well, and three out of the six neuropsychological measures sensitive to executive function displayed increases during the same interval period.

\section{Discussion}

Neuropathic pain is a very common pain condition experienced by the individuals with SCI. Gabapentin is being used frequently as the first drug of choice in SCI patients with neuropathic pain, but its adverse effect on cognition in this patient population has not been studied previously. In this study, we have found that gabapentin negatively impacts performance during testing of neuropsychological variables within one week of initiation of the medication. Without previous literature published on this population, and with decreases in the average score of six out of the nine neuropsychological tests in the 10 subjects at the 1 week post treatment assessment, we felt this warranted presentation of the preliminary results of this pilot study.

In comparison with the other medications used to treat neuropathic pain such as carbamazepine, valproic acid, and amytriptyline, gabapentin has been historically recognized to be well tolerated in general with a relatively mild side- 
Table 3 Average neuropsychological test score over 4 weeks

\begin{tabular}{llll}
\hline Tests & Baseline (SD) $^{\mathrm{a}}$ & 1 week (SD) & 4 weeks (SD) \\
\hline Digit Span Forwards (points) & $9.6(2.1)$ & $8.7(1.4)$ & $8.7(2.3)$ \\
Digit Span Backwards (points) & $5.6(1.8)$ & $6.4(1.5)$ & $5.5(2.8)$ \\
Mental Control (seconds) & $36.6(38.8)$ & $28.7(20)$ & $27(8.3)$ \\
Stroop test (seconds) & $13(2.1)$ & $13(3.2)$ & $11.7(3.1)$ \\
Word List: Immediate Recall (points) & $37.1(7.2)$ & $34.9(8.5)$ & $36.17(8.7)$ \\
Word List: Delayed Recall (points) & $7.4(2.7)$ & $5.6(3.3)$ & $7.5(3.1)$ \\
COWAT (points) & $41.6(12.7)$ & $35.4(11.8)$ & $32.3(11.2)$ \\
Oral Trail Making Test (seconds/errors) & $42.7(21)$ & $52.7(32.3)$ & $40.4(22.3)$ \\
Letter-Number Sequencing (points) & $9.7(2.8)$ & $8.9(3.1)$ & $10(3.3)$ \\
BPI: Pain Level (points) & $19.2(6.9)$ & $16.7(6)$ & $20.5(10.3)$ \\
BPI: Pain Interference (points) & $35.5(24.2)$ & $28.7(22.6)$ & $21(18.1)$ \\
\hline
\end{tabular}

${ }^{a}$ Scores listed in table represent the average score among the 10 subjects for each category

${ }^{\mathrm{b}}$ Values in table represent average score of 6 out of 10 patients owing to loss of follow-up
Table 4 Percentage changes in average scores of neuropsychological tests

\begin{tabular}{|c|c|c|}
\hline Tests & $\begin{array}{l}\text { Baseline vs } 1 \text { week } \\
\% \text { change }\end{array}$ & $\begin{array}{l}1 \text { week vs } 4 \text { week } \\
\% \text { change }^{\mathrm{a}}\end{array}$ \\
\hline Digit Span Forwards & $9.38 \%$ decrease & No change \\
\hline Digit Span Backwards & $14.29 \%$ increase & $14.06 \%$ decrease \\
\hline Mental Control & $21.58 \%$ decrease & $5.92 \%$ decrease \\
\hline Stroop Test & No change & $10.69 \%$ decrease \\
\hline $\begin{array}{l}\text { Word List: Immediate } \\
\text { Recall }\end{array}$ & $5.93 \%$ decrease & $3.63 \%$ increase \\
\hline $\begin{array}{l}\text { Word List: Delayed } \\
\text { Recall }\end{array}$ & $24.32 \%$ decrease & $33.93 \%$ increase \\
\hline COWAT & $14.9 \%$ decrease & $8.76 \%$ decrease \\
\hline Oral Trail Making Test & $23.42 \%$ increase & $23.34 \%$ increase \\
\hline $\begin{array}{l}\text { Letter-Number } \\
\text { Sequencing }\end{array}$ & $8.25 \%$ decrease & $12.36 \%$ increase \\
\hline BPI: Pain Level ${ }^{\mathrm{b}}$ & $13.02 \%$ decrease & $22.75 \%$ increase \\
\hline BPI: Pain Interference ${ }^{b}$ & $19.15 \%$ decrease & $26.83 \%$ increase \\
\hline
\end{tabular}

${ }^{a}$ Values represent average \% change of six patients owing to loss of follow-up of four patients after 1 week

${ }^{\mathrm{b}}$ Not considered a neuropsychological test

effect profile [16, 17]. Side effects that have been reported for gabapentin include: somnolence, dizziness, ataxia, fatigue, diarrhea, hypoventilation, visual field deficits, and sexual dysfunction [30, 31]. There are also reports of misusing and abusing gabapentin in up to $15 \%$ of patients [30]. In addition, several studies have indicated that gabapentin may also affect cognition [18, 19]. In a cross-over study comparing cognitive effects of carbamazepine vs gabapentin in healthy adults, Meador et al. [19] found that cognitive performance while taking gabapentin was statistically better than while taking carbamazepine in 8 out of 31 neuropsychological variables; however, when compared with performance while not taking either carbamazepine or gabapentin, four of the variables were significantly worse while on gabapentin. In another cross-over study, Meador et al. [19] compared the cognitive effects of carbamazepine vs gabapentin in healthy senior adults. When compared with the nondrug condition, both medications resulted in significantly worse performance on tests of verbal memory and motor speed, and participants reported a high incidence of sedative effects. Frequently reported side-effects for gabapentin included fatigue, unsteadiness, and dizziness. In addition, a study by Salinsky et al. [21] found that use of both carbamazepine and gabapentin resulted in EEG slowing in healthy volunteers, and they had significant effects on several objective and most subjective measures of mood and cognition.

In this study, gabapentin appears to negatively impact attention, memory, and general executive functioning in patients with SCI. Six out of the nine neuropsychological tests displayed a decrease when assessed 1 week after gabapentin treatment was initiated. The significance of these findings is perhaps more pronounced than the tables imply given that they are based on an equal expectation that the subjects would improve, decline, or remain unchanged. The expectation that the subjects would improve stems from the clinical perspective that most subjects would show some practice effect on some measures, resulting in a skewing toward improved performance over the trial period. At the time of admission, patients are also under a heavy stress burden owing to unfamiliar hospital environment, inexperience with injury, and potential lack of comfort with different modalities such as physical and occupational therapies. This may manifest as decreased baseline cognitive function values that improve as the patient becomes more acclimated with his/her surroundings. Instead, many neuropsychological measures exhibited decreased or stagnant performances at 1 week post treatment when compared with the baseline measurement, which may suggest that either initiation of gabapentin treatment and/or some other 
variable was negatively counteracting the benefit of practice effect and acclimation. An increase in five out of the nine tested neuropsychological variables at 4 weeks post treatment compared with 1 week post treatment suggests that the patients may have gotten used to the effects of gabapentin longer term. Once again, it is important to emphasize that the sample size was six individuals after the 1 week posttreatment assessment owing to loss of patient follow-up.

The association between pain and cognitive function is also worth discussing, as pain can act as a confounding variable in this study. It is hypothesized that pain competes with other attention-demanding stimuli, resulting in impaired cognitive domains such as attention and memory [10]. Complex tests of attention, such as those that involve interference or attention switching, may require executive function, and chronic pain patients perform poorly on such tests [10].

The average Pain Level score decreased from 19.2 to 16.7 (13.02\% decrease) from baseline to 1 week post treatment, whereas the average Pain Interference score decreased from 35.5 to 28.7 (19.15\% decrease). This improvement in pain levels with concurrent decline in various cognitive domains (six out of nine tests) suggests that pain is perhaps not the primary contributing cause of the observed decrease in cognitive results. However, given the small sample size and high drop-out rate, we could not conduct more detailed correlation of pain level and cognitive function.

Still, pain remains an under-reported, and under-treated problem across multiple venues of care. When the decision is being made whether or not to initiate gabapentin therapy, it is very important to adopt a shared decision-making approach that fosters discussion between the clinician and patient. Patients should be encouraged to weigh the possible benefits of gabapentin treatment against the potential consequences of sedation and cognitive decline in order to arrive at a decision that is appropriate for their particular plan of care.

Diminished cognition could have significant impact on one's ability to participate and learn during rehabilitation for SCI, as patients who struggle to follow and remember instructions from modalities such as physical or occupational therapies can experience prolonged hospital admissions. With the plethora of changes the patient is experiencing as a result of the injury, reducing their ability to process information, learn, and apply it could seriously impact the success of rehabilitation and their ability to transfer learned skills to the "outside world." This could ultimately impact long-term functional outcomes, independence, length of hospital stay, and community reintegration. Given the tangible effect that gabapentin appears to have on cognition, future studies should include control subjects with SCI who are not on gabapentin. Evaluation of the control group results will help determine whether some of the decreases in cognitive performance may be owing to the stresses of acute rehabilitation or other confounding factors such as other medications the patients may be on simultaneously. For example, seven of the subjects were also on baclofen and all subjects were on opioid medications, which may have also affected the cognitive function in these subjects. Although opioid effects on arousal and attention are well documented, there is conflicting evidence in the current literature regarding objective cognitive decline with opioid use. Despite subjective experiences of mental dullness and sedation, objective tests of cognitive functioning do not always demonstrate marked changes following opioid administration [32].

The results of this study may not be generalizable owing to the small sample size, loss of patient follow-up, lack of a control group, and inability to stratify patients to determine whether any decrease in cognitive function is attributable to gabapentin treatment versus other concurrently taken medications. However, the results of this pilot study can be used as justification to warrant future investigation, with the goal of raising further awareness regarding the potentially significant adverse effects of gabapentin. With a larger sample size, control group, and longer treatment duration, the results of a future study can provide statistically significant results that can influence the treatment regimen of SCI patients suffering from neuropathic pain. Furthermore, more recently, pregabalin is also being used for the treatment of neuropathic pain in individuals with SCI. Because of similarities of these two medications, future study should include subjects who are treated with pregabalin to track trends in cognitive performance.

\section{Conclusions}

In this study, gabapentin therapy was found to be associated with a decline in the average values of six out of the nine neuropsychological tests when measured at 1 week post initiation of gabapentin. Three out of the five tests sensitive to attention, three out of four sensitive to memory, and three out of the six tests sensitive to executive function displayed decreases in cognitive function at the 1 week post treatment assessment. Both measures of pain (level and interference) decreased during this interval. At the 4 week assessment post treatment, five out of the nine neuropsychological tests revealed an increase in cognitive function when compared with the 1 week post treatment values. Because of the lack of controls in this study, small sample size, and loss of patient follow-up, our results have limited generalizability, and we cannot definitely conclude that only the initiation of gabapentin caused the decline in cognitive performance. We feel that healthcare providers who are considering providing 
gabapentin to individuals with SCI for the treatment of neuropathic pain should be aware of potentially significant negative effect on cognitive function in these patients.

\section{Compliance with ethical standards}

Conflict of interest The authors declare that they have no conflict of interest.

\section{References}

1. Rintala DH, Loubser PG, Castro J, Hart KA, Fuhrer MJ. Chronic pain in a community-based sample of men with spinal cord injury: prevalence, severity, and relationship with impairment, disability, handicap, and subjective well-being. Arch Phys Med Rehabil. 1998;79:604-14.

2. Turner JA, Cardenas DD, Warms CA, McClellan CB. Chronic pain associated with spinal cord injuries: a community survey. Arch Phys Med Rehabil. 2001;82:501-9.

3. New PW, Lim TC, Hill ST, Brown DJ. A survey of pain during rehabilitation after acute spinal cord injury. Spinal Cord. 1997;35:658-63.

4. Werhagen L, Hultling C, Molander C. The prevalence of neuropathic pain after non-traumatic spinal cord lesion. Spinal Cord. 2007;45:609-15.

5. Finnerup NB, Johannesen IL, Sindrup SH, Bach FW, Jensen TS. Pain and dysesthesia in patients with spinal cord injury: a postal survey. Spinal Cord. 2001;39:256-62.

6. Siddall PJ, McClelland JM, Rutkowski SB, Cousins MJ. A longitudinal study of the prevalence and characteristics of pain in the first five years following spinal cord injury. Pain. 2003;103:249-57.

7. Treede RD, Jensen TS, Campbell JN, Cruccu G, Dostrovsky JO, Griffin JW, et al. Neuropathic pain: redefinition and a grading system for clinical and research purposes. Neurology. 2008;70:1630-5.

8. Cavenagh J, Good, Ravenscroft P. Neuropathic pain: are we out of the woods yet? Intern J Med. 2006;36:251-5.

9. Eide PK. Pathophysiological mechanisms of central neuropathic pain after spinal cord injury. Spinal Cord. 1998;36:601-12.

10. Moriarty O, Mcguire BE, Finn DP. The effect of pain on cognitive function: a review of clinical and preclinical research. Prog Neurobiol. 2011;93:385-404

11. Taylor CP, Gee NS, Su TZ, Kocsis JD, Welty DF, Brown JP, et al. A summary of mechanistic hypotheses of Gabapentin pharmacology. Epilepsy Res. 1998;29:233-49.

12. Putzke JD, Richards JS, Kezar L, Hicken BL, Ness TJ. Long-term use of Gabapentin for treatment of pain after traumatic spinal cord injury. Clin J Pain. 2002;18:116-21.

13. Levendoglu F, Ogun CO, Ozerbil O, Ogun TC, Ugurlu H. Gabapentin is a first line drug for the treatment of neuropathic pain in spinal cord injury. Spine. 2004;29:743-51.
14. Ahn SH, Park HW, Lee BS, Moon HW, Jang SH, Bae JH. Gabapentin effect on neuropathic pain compared among patients with spinal cord injury and different durations of symptoms. Spine. 2003;28:341-4.

15. Tai Q, Kirshblum S, Chen B, Millis S, Johnston M, DeLisa JA. Gabapentin in the treatment of neuropathic pain after spinal cord injury: A prospective, randomized, double-blind, crossover trial. J Spinal Cord Med. 2002;25:100-5.

16. To TP, Lim TC, Hill ST, Frauman AG, Cooper N, Kirsa SW, et al. Gabapentin for neuropathic pain following spinal cord injury. Spinal Cord. 2002;40:282-5.

17. Leach JP, Girvan J, Paul A, Brodie MJ. Gabapentin and cognition: a double blind, dose ranging, placebo controlled study in refractory epilepsy. J Neurol Neurosurg Psych. 1997;62:372-6.

18. Salinsky MC, Storzbach D, Spencer DC, Oken BS, Landry T, Dodrill CB. Effects of topiramate and Gabapentin on cognitive abilities in healthy volunteers. Neurology. 2005;64:792-8.

19. Meador KJ, Loring DW, Ray PG, Murro AM, King DW, Nichols ME, et al. Differential cognitive effects of carbamazepine and Gabapentin. Epilepsia. 1999;40:1279-85.

20. Martin R, Meador K, Turrentine L, Faught E, Sinclair K, Kuzniekcy $\mathrm{R}$, et al. Comparative cognitive effects of carbamazepine and Gabapentin in healthy senior adults. Epilepsia. 2001;42:764-71.

21. Salinsky MC, Binder LM, Oken BS, Storzbach D, Aron CR, Dodrill CB. Effects of Gabapentin and carbamazepine on the EEG and cognition in healthy volunteers. Epilepsia. 2002;43:482-90.

22. Mendoza T, Mayne T, Rublee D, Cleeland C. Reliability and validity of a modified Brief Pain Inventory short form in patients with osteoarthritis. Eur J Pain. 2006;4:353-61.

23. Plassman BL, Langa KM, Fisher GG, Heeringa SG, Weir DR, Ofstedal MB, et al. Prevalence of dementia in the United States: The aging, demographics, and memory study. Neuroepidemiology. 2007;29:125-32.

24. Wechsler D. WAIS-III Technical and Interpretive Manual. San Antonio, TX, USA: The Psychological Corporation; 2003.

25. Wechsler D. WMS-III Technical and Interpretive Manual. San Antonio, TX, USA: The Psychological Corporation; 2004.

26. Golden J. Stroop Color Word Test. Chicago, IL, USA: Soelting Co.; 1978.

27. Loonstra A, Tarlow A, Sellers AH. COWAT Metanorms across age, education, and gender. Appl Neuropsychol. 2001;8:161-6.

28. Barncord S. The Oral Trail Making Test: a validity investigation. Arch Clin Neuropsychol. 2002;17:827.

29. Strauss E, Sherman E, Spreen, Otfried. A. Compendium of Neuropsychological Tests. New York, NY, USA: Oxford University Press; 2006.

30. Quintero GC. Review about gabapentin misuse, interactions, contraindications and side effects. J Exp Pharmacol. 2017;9:13-21.

31. Kaufman KR, Struck PJ. Gabapentin-induced sexual dysfunction. Epilepsy Behav. 2011;21:324-6.

32. Ersek M, Cherrier MM, Overman SS, Irving GA. The cognitive effects of opioids. Pain Manag Nurs. 2004;5:75-78. 